\title{
Post-Finasteride Syndrome: An Induced Delusional Disorder with the Potential of a Mass Psychogenic Illness?
}

\author{
Ralph M. Trüeb ${ }^{a}$ Antonia Régnier ${ }^{a}$ Hudson Dutra Rezende ${ }^{a}$ \\ Maria Fernanda Reis Gavazzoni Dias ${ }^{\text {b }}$ \\ ${ }^{a}$ Center for Dermatology and Hair Diseases Professor Trüeb, Zurich-Wallisellen, Switzerland; ${ }^{b}$ Department of \\ Dermatology, Universidade Federal Fluminense, Centro de Ciências Médicas, Hospital Universitário Antonia Pedro, \\ Niterói, Brazil
}

\section{Established Facts}

- Finasteride represented a major breakthrough in the treatment of male pattern hair loss, based on an understanding of the underlying pathophysiology and observations on the respective genetic defect of 5-alpha-reductase.

- Clinical studies have demonstrated both a high efficacy of treatment and a very favorable safety profile, establishing the drug as first-line treatment of male pattern hair loss.

- More recently, post-finasteride syndrome (PFS) has been claimed to occur in men who have taken oral finasteride to treat either hair loss or benign prostatic hyperplasia. The condition is characterized by sexual dysfunction, somatic symptoms, and psychological disorders that persist after cessation of finasteride treatment.

\section{Novel Insights}

- We present the first case of PFS in our 20-year prescription practice of oral finasteride for treatment of male pattern baldness, with circumstantial evidence that PFS may represent a delusional disorder of the somatic type, possibly on a background of a histrionic personality disorder, and with the potential of a mass psychogenic illness due to its media coverage.

- PFS demonstrates analogies to controversial "mystery syndromes" as amalgam illness, multiple chemical sensitivity, Morgellons disease, and Koro: the symptoms cannot be adequately explained biologically, and the frequency of consultations for the respective condition parallels the media coverage, which points to a high degree of suggestibility.

- PFS has obviously become a problem that has to be dealt with, preferentially not on a nonprofessional public platform. There are no predictive factors for the risk of development of the PFS. Nevertheless, it would seem appropriate to ascertain a history of preexisting mental health issues, such as depression or histrionic personality disorder, with a strict selection of patients before starting treatment with finasteride. Also, management of PFS should be focused on the underlying psychopathological disorder.

\section{KARGER}

(c) 2019 S. Karger AG, Basel

E-Mail karger@karger.com

www.karger.com/sad
Prof. Ralph M. Trüeb, MD

Center for Dermatology and Hair Diseases Professor Trüeb Bahnhofplatz 1A

$\mathrm{CH}-8304$ Wallisellen (Switzerland)

E-Mail r.trueeb@ derma-haarcenter.ch 


\section{Keywords}

Post-finasteride syndrome · Finasteride - Dutasteride .

Delusional disorder of the somatic type $\cdot$ Histrionic personality disorder

\section{Abstract}

Post-finasteride syndrome (PFS) has been claimed to occur in men who have taken oral finasteride to treat either hair loss or benign prostatic hyperplasia, independent of age, dosage, or indication. By definition, the condition is characterized by sexual dysfunction, somatic symptoms, and psychological disorders that persist after cessation of finasteride treatment. As yet, the condition is not recognized by the medical community, although individuals who suffer from PFS present with relatively homogenous symptoms. The concept of PFS has emerged from reports of non-dermatologists, neuroendocrinological research and reflections, and uncontrolled studies of low quality and with a strong bias selection, while a significant nocebo effect among patients informed about possible side effects of finasteride is recognized. There are no predictive factors for the risk of development of PFS. Nevertheless, it has been suggested that a patient history of preexisting mental health disorder, particularly depression, may put patients at an increased risk. We report the first case of PFS in a long-standing (over 20 years) dermatotrichological practice with frequent finasteride prescription observed in a 25 -year-old male following dutasteride treatment for male androgenetic alopecia. There was circumstantial evidence that PFS may represent a delusional disorder of the somatic type, possibly on a background of a histrionic personality disorder, which would explain the refractoriness of the condition and a high degree of suggestibility.

(c) 2019 S. Karger AG, Basel

"I think of my body as a side effect of my mind"

Carrie Fisher (1956-2016)

Post-finasteride syndrome (PFS) has been claimed to occur in men who have taken oral finasteride to treat either hair loss or benign prostatic hyperplasia. By definition, the condition is characterized by sexual dysfunction, somatic symptoms, and psychological disorders that persist after cessation of finasteride treatment, independent of age, dosage, or indication [1-10]. As yet, the condition is not recognized by the medical community, although individuals who suffer from PFS present with relatively homogenous symptoms. Reported symptoms include loss of libido, erectile dysfunction, reduction in penis size and reduced sensation, gynecomastia, muscle atrophy, cognitive impairment, severely dry skin, and depression.

In 1992, $5 \mathrm{mg}$ oral finasteride was approved by the US Food and Drug Administration (FDA) for treatment of benign prostate hyperplasia, and in 1997, $1 \mathrm{mg}$ oral finasteride was approved for treatment of male pattern hair loss. Despite the long-standing and large-scale worldwide prescription of finasteride, PFS has only recently come into view attracting much attention in the media. The concept of PFS has emerged from reports of non-dermatologists, neuroendocrinological research and reflections, and uncontrolled studies of low quality and with a strong bias selection [1-10], while a significant nocebo effect among patients informed about possible side effects of finasteride is well recognized [11]. Ultimately, in 2012, a health advocacy group called the Post-Finasteride Syndrome Foundation was formed with the purpose of helping fund research on the characterization, underlying biologic mechanisms, and treatments of PFS, while improving public awareness of the condition.

While the true incidence of PFS as yet remains unknown, it has been estimated that over 1,000 men worldwide are experiencing the respective symptoms of PFS. This estimate is based on the number of registered users of the respective Internet forum www.propeciahelp.com.

A 2010 Cochrane review found that compared with placebo, men taking finasteride are at increased risk of impotence, erectile dysfunction, decreased libido, and ejaculation disorder for the first year of treatment; the rates of these effects became indistinguishable from placebo after 2-4 years and these side effects usually got better over time [12]. This is in line with the experience at the Center for Dermatology and Hair Diseases Professor Trüeb with a long-standing practice with frequent prescription of oral finasteride for successful treatment of male pattern hair loss.

We report the first case of PFS in our 20-year prescription practice of 5-alpha-reductase inhibitors for treatment of male pattern baldness, with circumstantial evidence that PFS may represent a delusional disorder of the somatic type, possibly on a background of a histrionic personality disorder.

\section{Case Report}

A 25-year-old Caucasian male was originally treated at the age of 20 years for male androgenetic alopecia Hamilton-Norwood III vertex with $5 \%$ topical minoxidil b.i.d. and later $10 \%$ topical minoxidil sulfate once daily for 4 years without success. Following the respective patient information on possible side effects of finaste- 
ride, $1 \mathrm{mg}$ oral finasteride was added to the topical minoxidil sulfate treatment at the age of 24 . After 7 months of combined $1 \mathrm{mg}$ oral finasteride and $10 \%$ topical minoxidil sulfate therapy, the result was still unsatisfactory, so that finasteride was switched to 0.5 mg oral dutasteride. During finasteride treatment, the patient did not report any side effects. Dutasteride was stopped after 1 month due to testicular tenderness. After cessation of dutasteride treatment, the testicular tenderness soon disappeared. Four months after cessation of a total duration of 8 months of 5-alpha-reductase inhibition with finasteride and dutasteride, respectively, the patient reported erectile dysfunction, sensation of cold in the penis, testicular pain, diminished volume of ejaculation, and loss of libido. At this time, the serum dihydrotestosterone level was decreased, but later normalized. No further endocrinological abnormalities were found.

From the beginning of treatments with finasteride and dutasteride, the patient and his mother repeatedly sent e-mails expressing their concerns and inquiring on the safety of the drugs and the labeling of persistent sexual side effects. With regard to the chronology of events surrounding the patient's ultimate complaint of suffering from PFS, it is noteworthy that it followed a health broadcasting on PFS on Swiss national television, in which an individual claimed suffering from the symptoms of the condition developing only after cessation of oral finasteride treatment for pattern hair loss. The patient also admitted to consulting respective Internet fora.

When the patient reported that he believed he was suffering from PFS, he was immediately called in for a consultation to check out the issue. Beforehand, he had consulted three urologists who did not find any organic or endocrinological abnormality explaining the symptoms reported by the patient. Throughout the conversation, the patient expressed his belief in suffering from PFS with unusual persistence, despite all rational argumentation against it, and was humorless and oversensitive about his concern. Every attempt to challenge his conviction aroused a strong emotional reaction, with irritability and hostility. He expressed a heightened sense of self-reference, and the atmosphere surrounding his belief was highly charged. He denied both a history of depression or a current depressive episode.

\section{Discussion}

After over 20 years of successful prescription practice of oral finasteride for treatment of male androgenetic alopecia, this is the first case of PFS seen at the Center for Dermatology and Hair Disease Professor Trüeb. It is noteworthy that repeated patient inquiries on safety issues of the drug, a broadcasting on PFS on Swiss national television, and respective Internet activities of the patient preceded his complaint.

In our opinion, PFS demonstrates some analogies to such controversial "mystery syndromes" as amalgam illness, multiple chemical sensitivity, Morgellons disease, and Koro for the following reasons: patients complain of symptoms that cannot be adequately explained biologi- cally, and the frequency of consultations for the conditions parallels the respective media coverage, which points to a high degree of suggestibility. Finally, the tenacity with which the patients hold on to their belief system, despite any rational argumentation against it, is indicative of at least a delusional aspect to their disorder.

Amalgam illness is the claim of a wide variety of debilitating and supposedly incurable conditions related to dental amalgam fillings and believed to be due to chronic mercury poisoning. In one study, mercury levels in blood and urine indeed correlated with the number of amalgam surfaces, consistent with the release of mercury from dental amalgam restorations. However, the mercury levels were far below those where negative health effects would be expected and were similar in patients with complaints self-related to dental amalgam restorations and healthy control individuals [13]. In another study, assays of mercury in urine samples of patients with amalgam illness indicated that the exposure was far below the levels at which symptoms could be indicated by psychometric tests. Psychologic investigation indicated that the symptoms were psychosomatic. All patients had experienced important psychic traumata in close correlation with the first appearance of symptoms [14].

Multiple chemical sensitivity is another disputed chronic condition characterized by typically vague and nonspecific symptoms that the affected individual attributes to low-level exposure to commonly used chemicals [15]. Although the symptoms themselves are tangible and can be disabling, the condition is not recognized as a somatic and chemical-induced illness by professional medical organizations. Blinded clinical trials show that individuals with multiple chemical sensitivity react as often and as strongly to placebo as they do to chemical stimuli, while the existence and severity of symptoms are related to the perception that a chemical stimulus is present [16]. Some experts assign the symptoms to either depression or somatoform or anxiety disorders [17].

Morgellons disease represents another form of self-diagnosed and unconfirmed condition in which individuals have skin lesions that they believe contain some kind of fibers [18]. Morgellons is poorly characterized, but the general medical consensus is that it is a form of delusional parasitosis, and the skin lesions are the result of compulsive scratching, while the fibers, when analyzed, turn out to originate from textiles. People usually self-diagnose Morgellons disease based on information from the Internet and find support and confirmation in online communities of people with similar illness beliefs. American medical sociologist and expert on mass psychogenic 
illness and hysteria Robert Bartholomew states that the "World Wide Web has become the incubator for mass delusion and Morgellons disease seems to be a socially transmitted disease over the Internet." According to this hypothesis, people with delusions of parasitosis and other psychological disorders become convinced after reading Internet accounts of others with similar symptoms. This is known as mass psychogenic illness, where physical symptoms without an organic cause spread to multiple people within the same community or social group [19].

Koro (Chinese: Suo yang) represents yet another peculiar delusional disorder with striking similarity to some of the alleged genital symptoms of PFS, such as penis shrinkage and diminished sensation. Koro is a culturebound delusional disorder in which an individual has an overpowering belief that the penis is shrinking, despite the lack of any true long-standing genital changes. The condition occurs worldwide, and mass hysteria of genital shrinkage anxiety has a history in Africa, Asia, and in the late Middle Ages in Europe [20]. The condition is listed in the Diagnostic and Statistical Manual of Mental Disorders and is commonly known as genital retraction syndrome in the United States and Europe. The Malleus Maleficarum, a 15th-century European manual for witchcraft investigations, relates stories of men claiming that their genitals had disappeared, being "hidden by the devil so that they can be neither seen nor felt" (Heinrich Kramer, Jakob Sprenger. Malleus Maleficarum. Part II, Question I, Chapter VII: How, as it were, they Deprive Man of his Virile Member). They were said to have reappeared after the men had appeased the responsible witches.

A delusional disorder is a primary mental illness characterized by the presence of unshakable beliefs in something not based on reality [21]. These delusions usually involve the misinterpretation of perceptions or experiences. In reality, however, the situations are either not true at all or highly exaggerated. Delusions can be bizarre in content, such as in Morgellons disease and Koro, or non-bizarre, such as in amalgam illness, multiple chemical sensitivity, and PFS. People with delusional disorder often can continue to socialize and function normally, apart from the subject of their delusion, and generally do not behave in an obviously odd manner. Unlike people with other psychotic disorders, who also might have delusions as a symptom of their disorder, patients with delusional disorder have no accompanying prominent hallucinations, thought disorder, mood disorder, or significant flattening of affect. The Diagnostic and Statistical Manual of Mental Disorders (DSM) defines several sub- types, of which in the somatic type, the person suffers from the delusion of having some physical defect or general medical condition.

Typically, the delusional patient expresses an idea or belief with unusual persistence or force, even when evidence suggests the contrary. The individual tends to be humorless and oversensitive about the belief. There is a quality of centrality: no matter how unlikely it is that these strange things are happening to them, the patient accepts them relatively unquestioningly. Every attempt to contradict the belief is likely to arouse an inappropriately strong emotional reaction, often with irritability and hostility. The delusion may lead to behaviors which are abnormal and/or out of character, although perhaps understandable in light of the delusional beliefs. Additional features include: it is a stable disorder characterized by the presence of delusions to which the patient clings with extraordinary tenacity; the illness is chronic and frequently lifelong; the delusions are logically constructed and internally consistent and do not interfere with general logical reasoning (although within the delusional system, the logic is perverted), and there is usually no general disturbance of behavior. If disturbed behavior does occur, it is directly related to the delusional beliefs. The individual experiences a heightened sense of self-reference. Events which, to others, are nonsignificant are of enormous significance to him, and the atmosphere surrounding the delusions is highly charged [22].

An induced delusional disorder is one in which symptoms of a delusional belief are transmitted from one individual to another. The same syndrome shared by more than two people (folie à deux) may be called folie à trois, à quatre, even à plusieurs, or ultimately folie "à l'Internet." Finally, when a large number of people may come to believe obviously false and potentially distressing things based purely on hearsay, these beliefs are labelled by the psychiatric profession as mass psychogenic illness or mass hysteria [23].

The high degree of suggestibility points to yet another possible underlying psychopathological disorder: histrionic personality disorder. Histrionic personality disorder is defined as a condition characterized by a pattern of excessive attention-seeking emotions, including an excessive need for approval. Histrionic people are lively, dramatic, vivacious, and enthusiastic. Histrionic personality disorder affects $2-3 \%$ of the general population. People with histrionic personality disorder have a high need for attention, make loud and inappropriate appearances, and exaggerate their behaviors and emotions. They express strong emotions with an impressionistic style and can be 
easily influenced by the suggestions of others. They have the propensity to somatization with neurological symptoms, which are not consistent with a well-established organic cause, the so-called conversion disorder, which was formerly known as hysteria. Conversion symptoms typically do not conform to known anatomical pathways and physiological mechanisms. It has sometimes been stated that the presenting symptoms tend to reflect the patient's own understanding of anatomy and that the less medical knowledge a person has, the more implausible are the presenting symptoms [24].

Maffei et al. [25] found the prevalence of personality disorders in subjects with male pattern hair loss to be significantly higher than in the general population, with three distinct personality profiles:

- Suspicious, with grandiose sense of self-importance, obsessive, and socially withdrawn.

- Impulsive, identity disordered, and socially maladjusted.

- Dramatic, emotional, and dependent.

Patients with personality disorders tend to be more difficult to handle with respect to treatment of their hair loss: nocebo reactions in particular are more frequent in patients with paranoid, passive-aggressive (negativistic), or histrionic personality disorders, while overvalued ideas are typical for patients with histrionic or narcissistic personality disorders [26].

Patients frequently become preoccupied with side effects when they are reluctant to undergo treatment, and some physicians also overestimate side effects [27]. Moreover, a significant nocebo effect has been demonstrated in patients who were informed of potential sexual adverse effects before taking finasteride versus patients who were not informed [28]. Of course, it is of utmost importance to inform patients of potential adverse effects, their frequencies, and appropriate management. Yet, the real concern should be the underlying medical condition, which is often displaced in the patient's mind by fear of the treatment. Patients must adopt a broader perspective, the long view, not a vision narrowed by fear. The physician's role is to help the patient figure out what he really wants and then to use the power of persuasion to show the patient the way there. At length, the way a physician phrases his recommendations can powerfully sway a patient's choice and have an influence on the treatment outcome [27]. Finally, the physician's choice has to be consistent with the patient's philosophy of living [27]. This particularly pertains to the prescription of oral finasteride for treatment of male pattern hair loss, where a choice must be made for long-term systemic medication with known and reversible (sexual side effects, gynecomastia, depression) and unknown risks (PFS, male infertility, prostate and breast cancer) for treatment of an essentially cosmetic condition.

In any case, PFS is obviously a problem that has to be dealt with, preferentially not on a public platform with the respective indiscriminate media coverage, which is in part propagated with primary commercial interests by providers of alternative hair growth treatments with inferior efficacy, such as low-level laser therapy, platelet-rich plasma, and mesotherapy.

While low-quality studies neither confirm nor refute PFS as a valid nosologic entity, it would be just as inappropriate to dismiss the condition as nonexistent as it is to demonize finasteride or dutasteride for treatment of male pattern hair loss. In fact, finasteride represented a major breakthrough in the treatment of male pattern hair loss, based on an understanding of the underlying pathophysiology [29] and observations on the respective genetic defect of 5-alpha-reductase [30]. Clinical studies have demonstrated both a high efficacy of treatment and a very favorable safety profile [31-38], establishing the drug as first-line treatment of male pattern hair loss. In the most recent study published in 2012, Sato and Takeda [38] reported on the efficacy and safety of $1 \mathrm{mg}$ oral finasteride for treatment of male pattern hair loss in the so far largest population study of 3,177 enrolled Japanese men. Efficacy was evaluated by global photographic assessment, and safety data were assessed by interviews and laboratory tests. The overall effect on hair growth was seen in $87.1 \%$, in whom hair increased greatly in $11.1 \%$, moderately in $36.5 \%$, and slightly in $39.5 \%$. The response rate improved with increasing duration of treatment. Adverse reactions occurred in $0.7 \%$ of men. Seven men discontinued treatment based on risk-benefit considerations. No specific safety problems associated with longterm use were observed. The authors concluded that in Japanese men with male pattern hair loss, $1 \mathrm{mg}$ oral finasteride used for long-term treatment maintains progressive hair regrowth without recognized side effect.

Eventually, dutasteride has been introduced for enhancement of efficacy in the treatment of male pattern hair loss due to its dual 5-alpha-reductase inhibition, therefore being capable of decreasing dihydrotestosterone levels to a greater extent than finasteride [39]. Respective clinical studies demonstrated superiority of 0.5 $\mathrm{mg}$ oral dutasteride versus $1 \mathrm{mg}$ oral finasteride in the treatment of male pattern hair loss $[40,41]$ and efficacy in men with male pattern hair loss recalcitrant to finasteride [42], while again being well tolerated. Since the plas- 
ma half-life time of dutasteride (3-5 weeks) is significantly longer than that of finasteride (age dependent, from 5-8 h), with respect to possible adverse effects related to therapeutic 5-alpha-reductase inhibition, it is advisable to start patients on oral finasteride and to switch from finasteride to dutasteride only if results are unsatisfactory at 6 months and tolerance is good.

Up to date, there are no predictive factors for the risk of development of PFS. Nevertheless, it would seem appropriate to ascertain a history of preexisting mental health issues, such as depression or histrionic personality disorder, with a strict selection of patients before starting treatment with finasteride, since these may put patients at an increased risk of developing emotional disorders with somatization related to 5-alpha-reductase inhibition therapy [43].

In any case of adverse effects [44], oral finasteride or dutasteride treatment should immediately be stopped. Since no evidence of androgen deficiency, persistent steroid 5-alpha-reductase inhibition, or androgen insensi- tivity have been found so far, patients with PFS are unlikely to benefit from treatment with testosterone, dihydrotestosterone, or any other androgen. Attention must be focused rather on the treatment of associated specific psychopathological disorders and related sexual symptoms with appropriate psychotherapy and specific psychotropic agents depending on the type of the underlying mental disorder (depressive, delusional, or somatoform disorder) [45].

\section{Statement of Ethics}

The authors have no ethical conflicts to disclose. The indicator patient has given his written consent to publish details of the case.

\section{Disclosure Statement}

The authors declare no conflicts of interest.

\section{References}

1 Gormley GJ, Stoner E, Bruskewitz RC, Imperato-McGinley J, Walsh PC, McConnell JD, et al.; The Finasteride Study Group. The effect of finasteride in men with benign prostatic hyperplasia. N Engl J Med. 1992 Oct;327(17): 1185-91.

2 Traish AM, Haider KS, Doros G, Haider A Finasteride, not tamsulosin, increases severity of erectile dysfunction and decreases testosterone levels in men with benign prostatic hyperplasia. Horm Mol Biol Clin Investig. 2015 Sep;23(3):85-96.

3 Traish AM, Hassani J, Guay AT, Zitzmann M, Hansen ML. Adverse side effects of $5 a-$ reductase inhibitors therapy: persistent diminished libido and erectile dysfunction and depression in a subset of patients. J Sex Med. 2011 Mar;8(3):872-84.

4 Irwig MS, Kolukula S. Persistent sexual side effects of finasteride for male pattern hair loss. J Sex Med. 2011 Jun;8(6):1747-53.

5 Irwig MS. Persistent sexual side effects of finasteride: could they be permanent? J Sex Med. 2012 Nov;9(11):2927-32.

6 Di Loreto C, La Marra F, Mazzon G, Belgrano E, Trombetta C, Cauci S. Immunohistochemical evaluation of androgen receptor and nerve structure density in human prepuce from patients with persistent sexual side effects after finasteride use for androgenetic alopecia. PLoS One. 2014 Jun;9(6):e100237.
7 Ganzer CA, Jacobs AR, Iqbal F. Persistent sexual, emotional, and cognitive impairment postfinasteride: a survey of men reporting symptoms. Am J Men Health. 2015 May;9(3):222-8.

8 Ali AK, Heran BS, Etminan M. Persistent sexual dysfunction and suicidal ideation in young men treated with low-dose finasteride: a pharmacovigilance study. Pharmacotherapy. 2015 Jul;35(7):687-95.

9 Irwig MS. Depressive symptoms and suicidal thoughts among former users of finasteride with persistent sexual side effects. J Clin Psychiatry. 2012 Sep;73(9):1220-3.

10 Altomare G, Capella GL. Depression circumstantially related to the administration of finasteride for androgenetic alopecia. J Dermatol. 2002 Oct;29(10):665-9.

11 Fertig R, Shapiro J, Bergfeld W, Tosti A. Investigation of the Plausibility of 5-Alpha-Reductase Inhibitor Syndrome. Skin Appendage Disord. 2017;2(3-4):120-129

12 Tacklind J, Fink HA, Macdonald R, Rutks I, Wilt TJ. Finasteride for benign prostatic hyperplasia. Cochrane Database Syst Rev. 2010 Oct;2010(10):CD006015.

13 Bratel J, Haraldson T, Meding B, Yontchev E, Ohman SC, Ottosson JO. Potential side effects of dental amalgam restorations. (I). An oral and medical investigation. Eur J Oral Sci. 1997 Jun;105(3):234-43.

14 Lindberg NE, Lindberg E, Larsson G. Psychologic factors in the etiology of amalgam illness. Acta Odontol Scand. 1994 Aug;52(4): 219-28.
15 Genuis SJ. Chemical sensitivity: pathophysiology or pathopsychology? Clin Ther. 2013 May;35(5):572-7.

16 Bornschein S, Hausteiner C, Römmelt $\mathrm{H}$, Nowak D, Förstl H, Zilker T. Double-blind placebo-controlled provocation study in patients with subjective Multiple Chemical Sensitivity (MCS) and matched control subjects. Clin Toxicol (Phila). 2008 Jun;46(5):443-9.

17 Bornschein S, Förstl H, Zilker T. Idiopathic environmental intolerances (formerly multiple chemical sensitivity) psychiatric perspectives. J Intern Med. 2001 Oct;250(4):309-21.

18 Vulink NC. Delusional Infestation: state of the Art. Acta Derm Venereol. 2016 Aug; 96(217):58-63.

19 Bartholomew R, Hassall P. A Colorful History of Popular Delusions. Prometheus Books; 2015. ISBN: 1633881229

20 Mattelaer JJ, Jilek W. Koro-the psychological disappearance of the penis. J Sex Med. 2007 Sep;4(5):1509-15.

21 American Psychiatric Association. Diagnostic and Statistical Manual of Mental Disorders. 5th ed., text revision. Washington (DC): American Psychiatric Association; 2013.

22 Munro A. Delusional disorder: paranoia and related illnesses. Cambridge, UK: Cambridge University Press, 1999. ISBN 0-521-58180-X. https://doi.org/10.1017/CBO9780511544095.

23 Bartholomew RE, Wessely S. Protean nature of mass sociogenic illness: from possessed nuns to chemical and biological terrorism fears. $\mathrm{Br} \mathrm{J}$ Psychiatry. 2002 Apr;180(04):300-6. 
24 American Psychiatric Association. Diagnostic and Statistical Manual of Mental Disorders DSM-IV-TR. Chapter 16: Personality Disorders. American Psychiatric Publishing. 2000

25 Maffei C, Fossati A, Rinaldi F, Riva E. Personality disorders and psychopathologic symptoms in patients with androgenetic alopecia. Arch Dermatol. 1994 Jul;130(7):868-72.

26 Trüeb RM. Psychopathological Disorders: In: Trüeb RM. The Difficult Hair Loss Patient. Guide to Successful Management of Alopecia and Related Conditions. Springer 2015: p. 168 Groopman, J. How Doctors Think. Boston, New York: Houghton Mifflin Company; 2007

27 Mondaini N, Gontero P, Giubilei G, Lombardi G, Cai T, Gavazzi A, et al. Finasteride $5 \mathrm{mg}$ and sexual side effects: how many of these are related to a nocebo phenomenon? J Sex Med. 2007 Nov;4(6):1708-12.

28 Kaufman KD. Androgen metabolism as it affects hair growth in androgenetic alopecia. Dermatol Clin. 1996 Oct;14(4):697-711.

29 Imperato-McGinley J, Guerrero L, Gautier T, Peterson RE. Steroid 5alpha-reductase deficiency in man: an inherited form of male pseudohermaphroditism. Science. 1974 Dec; 186(4170):1213-5.

30 Kaufman KD, Olsen EA, Whiting D, Savin R, DeVillez R, Bergfeld W, et al.; Finasteride Male Pattern Hair Loss Study Group. Finasteride in the treatment of men with androgenetic alopecia. J Am Acad Dermatol. 1998 Oct;39(4 Pt 1):578-89.

31 Leyden J, Dunlap F, Miller B, Winters P, Lebwohl M, Hecker D, et al. Finasteride in the treatment of men with frontal male pattern hair loss. J Am Acad Dermatol. 1999 Jun;40(6 Pt 1):930-7.
32 Whiting DA, Waldstreicher J, Sanchez M, Kaufman KD. Measuring reversal of hair miniaturization in androgenetic alopecia by follicular counts in horizontal sections of serial scalp biopsies: results of finasteride $1 \mathrm{mg}$ treatment of men and postmenopausal women. J Investig Dermatol Symp Proc. 1999 Dec; 4(3):282-4.

33 Van Neste D, Fuh V, Sanchez-Pedreno P, Lopez-Bran E, Wolff H, Whiting D, et al. Finasteride increases anagen hair in men with androgenetic alopecia. Br J Dermatol. 2000 Oct; 143(4):804-10.

34 Finasteride Male Pattern Hair Loss Study Group. Long-term (5-year) multinational experience with finasteride $1 \mathrm{mg}$ in the treatment of men with androgenetic alopecia. Eur J Dermatol. 2002 Jan-Feb;12(1):38-49.

35 Kawashima M, Hayashi N, Igarashi A, Kitahara H, Maeguchi M, Mizuno A, et al. Finasteride in the treatment of Japanese men with male pattern hair loss. Eur J Dermatol. 2004 Jul-Aug;14(4):247-54.

36 Price VH, Menefee E, Sanchez M, Kaufman KD. Changes in hair weight in men with androgenetic alopecia after treatment with finasteride (1 mg daily): three- and 4-year results. J Am Acad Dermatol. 2006 Jul;55(1): 71-4.

37 Kaufman KD, Rotonda J, Shah AK, Meehan AG. Long-term treatment with finasteride 1 mg decreases the likelihood of developing further visible hair loss in men with androgenetic alopecia (male pattern hair loss). Eur J Dermatol. 2008 Jul-Aug; 18(4):400-6.

38 Sato A, Takeda A. Evaluation of efficacy and safety of finasteride $1 \mathrm{mg}$ in 3177 Japanese men with androgenetic alopecia. J Dermatol. 2012 Jan;39(1):27-32.
39 Olsen EA, Hordinsky M, Whiting D, Stough D, Hobbs S, Ellis ML, et al.; Dutasteride Alopecia Research Team. The importance of dual 5alpha-reductase inhibition in the treatment of male pattern hair loss: results of a randomized placebo-controlled study of dutasteride versus finasteride. J Am Acad Dermatol. 2006 Dec;55(6):1014-23.

40 Eun HC, Kwon OS, Yeon JH, Shin HS, Kim BY, Ro BI, et al. Efficacy, safety, and tolerability of dutasteride $0.5 \mathrm{mg}$ once daily in male patients with male pattern hair loss: a randomized, double-blind, placebo-controlled, phase III study. J Am Acad Dermatol. 2010 Aug;63(2):252-8.

41 Gubelin Harcha W, Barboza Martínez J, Tsai TF, Katsuoka K, Kawashima M, Tsuboi R, et al. A randomized, active- and placebo-controlled study of the efficacy and safety of different doses of dutasteride versus placebo and finasteride in the treatment of male subjects with androgenetic alopecia. J Am Acad Dermatol. 2014 Mar;70(3):489-498.e3.

42 Jung JY, Yeon JH, Choi JW, Kwon SH, Kim BJ, Youn SW, et al. Effect of dutasteride $0.5 \mathrm{mg} / \mathrm{d}$ in men with androgenetic alopecia recalcitrant to finasteride. Int J Dermatol. 2014 Nov;53(11):1351-7.

43 Ganzer CA, Jacobs AR. Emotional consequences of finasteride: fool's gold. Am J Men Health. 2018 Jan;12(1):90-5.

44 Trüeb RM. Tackling Adverse Effects. In: Trüeb RM, editor. The Difficult Hair Loss Patient. Guide to Successful Management of Alopecia and Related Conditions. Springer; 2015. p. 181

45 Basaria S, Jasuja R, Huang G, Wharton W, Pan $\mathrm{H}$, Pencina K, et al. Characteristics of men who report persistent sexual symptoms after finasteride use for hair loss. J Clin Endocrinol Metab. 2016 Dec;101(12):4669-80. 